\title{
SAV Operations on a Bus Line Corridor: Travel Demand, Service Frequency, and Vehicle Size
}

\author{
Huang Yantao, ${ }^{1}$ Kara M. Kockelman $\mathbb{D}^{1},{ }^{1}$ and Long T. Truong $\mathbb{D}^{2}$ \\ ${ }^{1}$ Department of Civil, Architectural and Environmental Engineering, The University of Texas, Austin, TX, USA \\ ${ }^{2}$ Department of Engineering, La Trobe University, Melbourne, Australia \\ Correspondence should be addressed to Kara M. Kockelman; kkockelm@mail.utexas.edu
}

Received 23 February 2021; Revised 27 May 2021; Accepted 26 June 2021; Published 13 July 2021

Academic Editor: Jing Zhao

Copyright (C) 2021 Huang Yantao et al. This is an open access article distributed under the Creative Commons Attribution License, which permits unrestricted use, distribution, and reproduction in any medium, provided the original work is properly cited.

Before shared automated vehicles (SAVs) can be widely adopted, they are anticipated to be implemented commercially in confined regions or fixed routes where the benefits of automation can be realized. SAVs have the potential to operate in a traditional transit corridor, replacing conventional transit vehicles, and have frequent interactions with riders and other vehicles sharing the same right of way. This paper microsimulates SAVs' operation on a 6.5-mile corridor to understand how vehicle size and attributes of such SAV-based transit affect traffic, transit riders, and system costs. The SUMO (Simulation of Urban MObility) platform is employed to model microscopic interactions among SAVs, transit passengers, and other traffic. Results show that the use of smaller, but more frequent, SAVs leads to reduced passenger waiting times but increased vehicle travel times. More frequent services of smaller SAVs do not, in general, significantly affect general traffic due to shorter dwell times. Overall, using smaller SAVs instead of the large 40 -seat SAVs can reduce system costs by up to $4 \%$ while also reducing passenger waiting times, under various demand levels and passenger loading factors. However, the use of 5-seat SAVs does not always have the lowest system costs.

\section{Introduction}

Automated vehicles (AVs) and shared mobility will fundamentally change the future traffic pattern, by providing cost, environmental, and safety benefits [1-4]. Shared AVs (SAVs) offer more potential benefits through a lower-cost on-demand service that can be flexible in both schedules and routes [5]. Currently, SAV tests are being performed worldwide, as people try to envision the emerging service format that SAVs can offer in both the near and far future [6]. Over 40 corporations are making an effort to bring AVs [7] into the automobile market. For example, Waymo [8] has tested its AVs in Arizona and Texas and achieved 4 million self-driven miles by November 2017. NAVYA [9] had over 130 automated shuttles running worldwide in 2019 in 7 types of places (city, airport, campus, hospital, resort, theme park, and industry), including Mcity in Michigan and Lake Nona in Orlando.

As seen from existing pilot AV tests, SAVs are anticipated to be implemented commercially in a confined region where full-automation benefits can be realized first, before they can run everywhere. University campuses and central business areas are candidate locations for early SAV implementation [10], and SAVs are also likely to provide first-mile last-mile service near transit stations [11]. This paper investigates another potential service that SAVs can provide, which is replacing the role of 40 -seater buses in a traditional transit corridor. Attention to such service transformations will grow as the automation technology matures, enabling SAVs to manage frequent and complex interactions with riders and other vehicles.

SAVs can serve as transit vehicles, with fixed or variable routes, stop locations, and frequencies. They can be a mixture of different sizes, depending on demand, from a 4-seater (much like the common sedan, with ridesharing en route) to 10-passenger vehicles (an automated van), 20-passenger vehicles (an automated shuttle or minibus), and vehicles that can carry 40 or more passengers (an automated full-size bus). Smaller SAVs (like 4- or 6-seat vehicles) are nimbler and 
easier to park, can accelerate faster, and may cause less congestion and sightline issues. They are capable of running flexible routes for point-to-point on-demand service and are able to provide fixed-route service, where they often skip stops because of fewer boardings and alightings. A large fleet size of small-size SAVs, however, may be required to maintain a high-frequency service compared to large-size SAVs. On the contrary, large SAVs usually run fixed routes with a relatively small fleet size and can be more space-efficient (per personmile traveled) but will have to stop more often at stations, causing delays along the corridor.

Extensive ongoing studies are investigating what changes SAVs would bring to the environment, urban congestion, and shared economy [12-14], mostly through door-to-door service; however, there is a lack of research about the impact of various vehicle sizes on SAV-transit service under different travel demand levels, considering discrepancies in stop-skipping behavior, SAVs' interactions between riders and other vehicles, and the network performance (e.g., riders' wait time, vehicle delay, etc.). Seeking the best SAV size for a transit corridor not only relates to the riders but is also important to the service provider. Riders would like to experience less waiting time and onboard time with fewer stops and rerouting, while SAV operators would like to maximize profit or social welfare. Therefore, the objective of this study is to understand how traffic reacts to and how riders and system costs are affected by vehicle sizes and performance attributes for SAV-based "transit," with hypothesis tests on whether smaller AVs offer better overall service to corridor travelers. Various scenarios are set up to microsimulate SAVs' operation in a 6.5-mile corridor, including different road congestion status, adding traffic signals for the corridor and adding parking bays for SAVs' stops.

The paper is arranged as follows. The next section reviews current studies about leveraging SAV simulations to understand the service performance of the fleet, followed by methodologies about the detailed proposed simulation model for the SAV-transit service. After introducing the setups of the scenario, results and analysis are presented. The last section concludes this study and proposes future work.

\section{Literature Review}

SAV simulation efforts are usually made for door-to-door service, around fleet sizing decisions [13-15], ridesharing mechanisms [16], electric vehicles involving charging decisions $[17,18]$, and environmental effects [2, 12]. Spieser et al. [14] investigated the proper fleet size in Singapore that could serve the travel demand while ensuring a desired level of service. Their results showed that an SAV fleet size of about one-third of the total number of passengers was desired in Singapore. A one SAV per 9.3 conventional vehicles replacement was shown in Fagnant et al.'s [15] simulation in the Austin area. Recent fleet sizing decision studies mostly assume that an SAV has a maximum occupancy of 4 passengers; however, current SAVs used for the tests can usually hold more than 4 passengers and are expected to accommodate 20 or more passengers at maximum [19].
Microsimulation noted in this paper refers to the microscopic model in traffic flow, where individual driving behavior is tracked, like detailed car-following and lanechanging maneuvers. This is different from many mesoscopic simulations, where vehicles' performance attributes (e.g., acceleration, deceleration, and headway) are not usually tracked and vehicles' lane changing is also ignored when vehicles are traveling on a link (or roadway). Mesoscopic simulations provide valuable results in regional fleet sizing decisions, mechanisms of ridesharing or even dynamic ridesharing, and traffic patterns under dynamic traffic assignment, but they are not able to capture multimodal interactions at the microscopic level between SAVs and conventional vehicles, such as at stations. While this is easier for microscopic simulations, SAV microsimulation studies are not often seen.

Alozi and Hamad [20] used VISSIM to microsimulate connected AVs (CAVs) on a 7-kilometer freeway segment in Dubai. Results from various CAV market penetrations were compared, in terms of vehicle delay, speed, and travel time. Since VISSIM provides adjusted car-following and lanechanging models to accommodate CAV features [20], these models were applied directly. Authors observed an $86 \%$ decrease in delay under a $100 \%$ CAV penetration scenario and results showed that the greatest benefits of CAVs are obtained when market penetration of CAVs ranges from $0 \%$ to $20 \%$ and from $70 \%$ to $80 \%$. However, this is a simulation study that investigates the impacts of personally owned CAVs only, as SAVs (shared ride) are not considered. Also through VISSIM software, Guo et al. [21] investigated how information-based managed lane choice decisions would impact the traffic performance, under a mixture of CAVs and human-driven vehicles. Zhu et al. [10] quantified the mobility and energy benefits of SAVs running a fixed route in a small network. By leveraging the Simulation of Urban Mobility (SUMO) platform that is capable of real-time microsimulation control of passengers and vehicles, SAVs can take ridesharing requests and pick up and drop off passengers through a dispatching pattern. Huang et al. [11] further microsimulated a $3 \mathrm{mi} \times 6 \mathrm{mi}$ central Austin area with SAVs providing first-mile last-mile service to Austin's Red Line commuter rail stations. VMT was predicted to rise $3.7 \%$ in central Austin with average vehicle occupancy falling $30 \%$ (from 1 to 0.74 people per vehicle), due to empty SAV driving (between riders).

Some other studies investigate the integration of SAV and transit use [22-25], but they are not at the microscopic level. Shen et al. [24] simulated an integrated AV and public transportation system based on Singapore's transit structure and demand characteristics. An agent-based supply-side simulation was built to assess the performance of the proposed service with different fleet sizes and ridesharing preferences in Singapore's $12 \mathrm{~km}^{2}$ area during morning peak hours from 7 am to $9 \mathrm{am}$. Authors showed that the integrated system has the potential of enhancing service quality, occupying fewer road resources, being financially sustainable, and utilizing bus services more efficiently. Wen et al. [25] investigated the opportunities of AVs and public transit in a major European city using static-travel-time agent- 
based simulation. They simulated scenarios with various fleet sizes, vehicle capacities (up to 4 passengers), fare schemes, and hailing strategies only for the connections to the transit station. A nested logit mode choice model was presented, considering 4 modes (bus, rail, park-and-ride, and AV-andride) nested under transit mode. It was reported that 560 vehicles can accommodate the travel demand in the city if sharing is not available, but the fleet size can be reduced to fewer than 200 vehicles if an SAV can be shared by 4 people.

Overall, there has been extensive research dedicated to understanding the future travel pattern with automation technology. The focus of mesoscopic simulation is unilaterally on the desired vehicle fleet size to meet door-to-door travel demand, or the integrated system of SAV and transit, with a simple car-following model and no lane changing behavior. At the microscopic level, efforts have been dedicated to the interaction between AVs and human-driven vehicles, lacking other interactions that may exist (e.g., riders' boarding and alighting, parking on the road, etc.). Planning for the emerging transformation of public transportation is via evaluating SAV-transit service requests for the integration of passenger and vehicle behavior at the microscopic level and an investigation of all the service levels that SAVs can provide through different vehicle attributes. Filling the gaps of existing studies, this study contributes by (1) envisioning a future transit corridor affected by SAVbased "transit," (2) microsimulating the detailed behavior of passengers and vehicles, and (3) conducting cost analysis of such a transit service to provide insights on future transit planning under emerging technologies.

\section{Microsimulation Description}

3.1. SUMO Simulation Setup. This study uses SUMO software, which can microsimulate interactions among different travel modes [26]. The detailed manipulations are achieved through TraCI (Traffic Control Interface), a toolkit in SUMO that allows for the retrieval of real-time values of simulated objects, which can conduct user-defined behavior "online" through Python scripts.

The simulation network setup in this study is a 6.5-mile, 2-lane, straight corridor with no traffic signals. This corridor has a lane width of $11.6 \mathrm{ft}$ and a speed limit of $30 \mathrm{mph}$ based on recommended designing practice from the American Public Transportation Association [27]. As also suggested in previous studies, SAV stops are evenly placed (every quarter mile) along the corridor [28] at the middle point of a road segment. Two types of vehicles, namely SAVs and conventional vehicles (referred to as background flow in the following context), can travel in both lanes. SAVs are inserted into the corridor to serve riders with a fixed schedule (i.e., a fixed frequency) and run a fixed route to the end of the corridor, with stops at the stations if necessary. A Poisson distributed background flow of conventional cars with a mean departure rate is assumed. The base case scenario will test a departure rate of 0.7 vehicles per second, which is approximately $1260 \mathrm{vphpl}$ (vehicle per hour per lane), to reflect a common flow rate on a 30-mph corridor [27]. Due to curbside stops, SAVs obstruct the vehicles behind when serving passengers at stops. Scenarios that have parking bays and added traffic signals for SAVs are also tested in a later context. With a high dispatching rate (frequency) of SAVs, "bus bunching" can be observed, as SAVs can overtake previously dispatched SAVs.

Travel demand in this study refers to SAV riders/passengers, who walk on the road (access or egress the SAV stops), wait for SAVs, and ride in SAVs. Other active modes are ignored here because they hardly affect the operations of SAVs or conventional vehicles, although there may be taxis, which will stop and pick-up/drop-off passengers, and scooters/bicycles, which could potentially slow down the traffic. Passengers are uniformly generated at random along the corridor, arriving with a uniform distribution in a 3 hour period of peak time. Since the distance between two consecutive stations is $1 / 4$ miles, passengers who have origins and destinations between two consecutive stations probably give up taking transit. Therefore, only those passengers who have a trip distance longer than $1 / 3$ miles are considered rational SAV users and randomly generated along the corridor. These riders walk to the nearest stop, get on the next available SAV, and get off at the stop closest to his/her destination. An SAV that has not reached its capacity will stop at a stop where new riders are waiting or where current riders want to alight. Further, an SAV waits for passengers approaching the stop if this SAV has stopped at the station and the passenger can walk to catch this SAV in 10 seconds. After a rider gets off the SAV, he/she walks to the destination location and then disappears from the simulator. Figure 1 is an illustration of the SAV bus line corridor. One passenger is shown to be waiting at the stop, while the SAV has just arrived at that stop, causing the following vehicles to brake.

\subsection{Simulation Framework. Figure 2 shows the simulation} design details, by demonstrating the whole simulation flow of how vehicles and riders interact in the simulator. Road network and bus stop information are read first, including the edge lengths as well as the length and position of the SAV stops. Travel demand is then processed to obtain the start location of the journey, departure station, arrival station, and destination location of the itinerary. After that, the simulation starts, and the itinerary of riders is processed by the simulator at time 0 . Beginning at the first step of the simulation, background flow comes into the corridor and SAVs depart from the start point of the corridor based on a fixed headway. During every step of the simulation, which is every 0.5 seconds, the status of riders and SAVs is tracked and updated. Riders' status is tracked to inform SAVs (e.g., whether riders are catching the SAV, waiting at the bus station, or already on board) so that SAVs can react accordingly. The status and locations of SAVs are also tracked, to determine whether the SAVs need to stop at the next station. The designed simulation also checks whether riders need to get off at the next station. If no one is getting off, it then checks whether passengers are waiting at the next station and if there are available seats on board. When the SAV is dwelling at a stop, it keeps checking whether a rider can catch the SAV in 10 seconds before its departure and will 


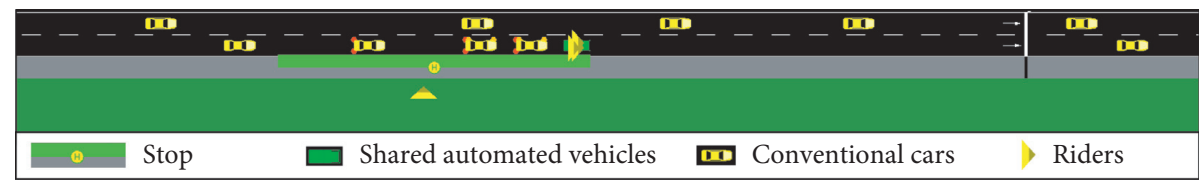

FIgURE 1: Bus line simulation.

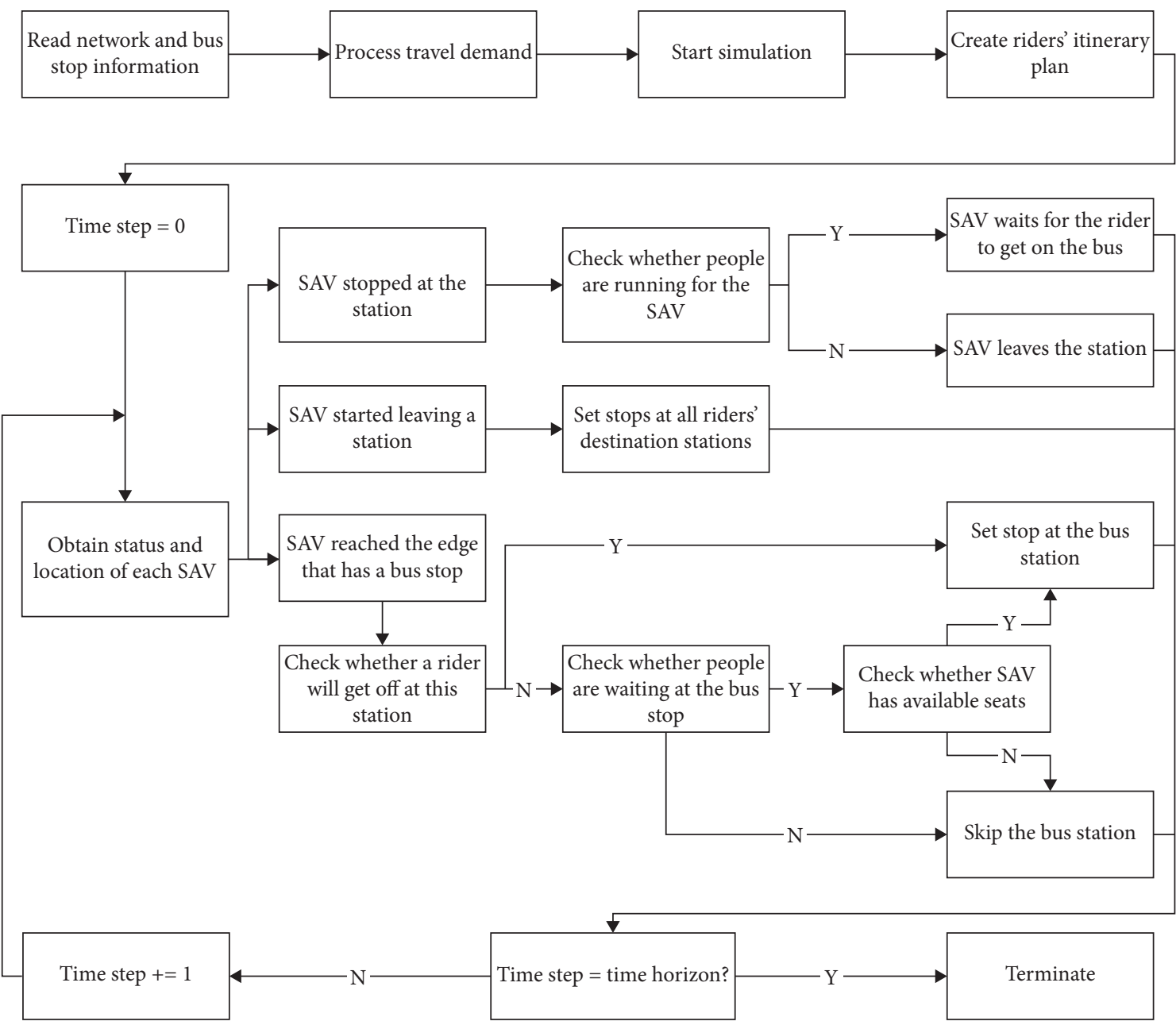

FIgURE 2: Simulation design.

then let those people get on the SAV. After the SAV leaves the station, it sets future stops for all new riders' destination stations. SAVs' and riders' status and locations are checked every timestep until the simulation reaches the time horizon. The output includes both passenger and vehicle information, such as vehicles' and riders' travel time and waiting time, background vehicle speed, and average vehicle occupancy.

3.3. Simulation Parameters. Stocker and Shaheen [19] have envisioned four types of potential SAV and service models: microvehicles (1 or 2 passengers), small vehicles (3-7 passengers), mid-sized vehicles (7-20 passengers), and large vehicles (20+ passengers). However, one can imagine that the future world may also have a large capacity SAV - an automated bus to serve a heavy demand transit corridor, but such a corridor probably does not allow microvehicles to travel. Therefore, for the simplicity of vehicle sizes, four types of vehicles are simulated, from a normal sedan size of 5 seats (no driver due to full automation) to an automated bus of 40 seats.

As shown in Table 1, background flow uses the SUMO default value for "passenger" vehicle types [26]. Although there would be differences in the lane-changing model between conventional vehicles, this study focuses on longitudinal effects instead of lateral effects. Therefore, the lane-changing model is assumed to be LC2013, the default from SUMO [29]. The dimensions of different SAV sizes are determined by referring to existing vehicle sizes (Table 1 ), although one may expect a different interior design for SAVs. Without a driver's seat, space in SAVs can be better utilized and standing may even be allowed in smaller size SAVs. 
TABLE 1: Vehicle configurations and other simulation parameters.

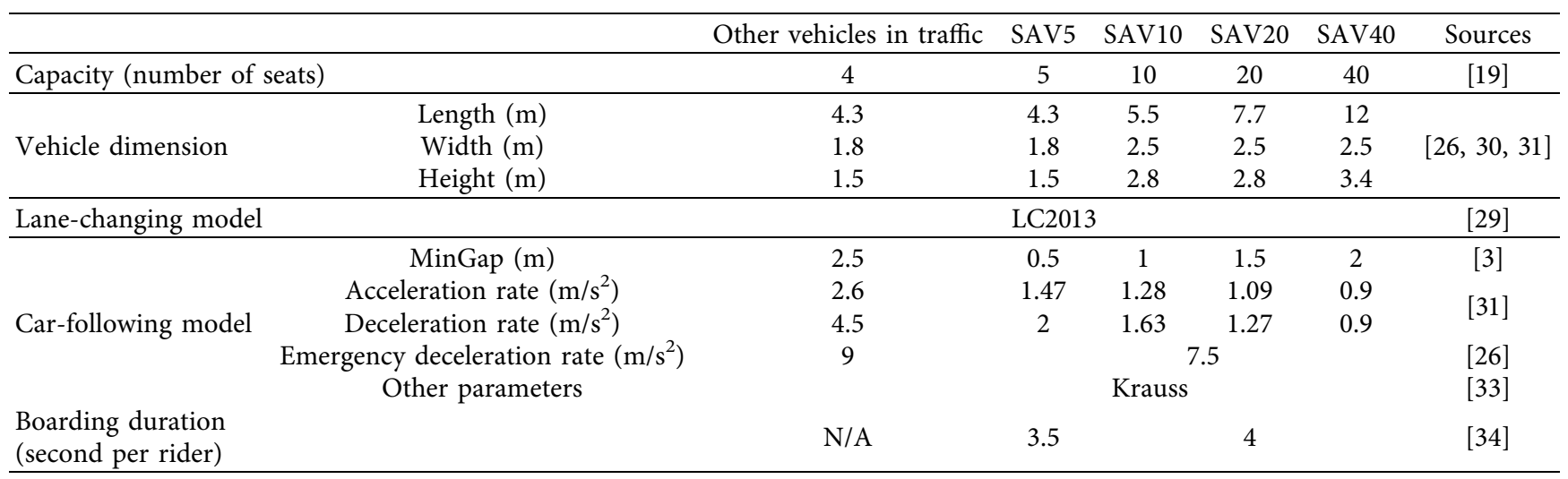

Automated bus or shuttle tests may proceed with caution at early implementation stages due to the unreliable and unstable camera recognition and slow data processing; however, in the future, automated buses will probably have faster speeds than human-driven vehicles [35]. In terms of transit operations, comfort is also key to the design of vehicles, based on the study from Bae et al. [32], who did a summary of the possible range for acceleration and deceleration rates of a comfort transit vehicle. Since small AVs are nimble and can have faster acceleration and deceleration rates, due to the use of the safety belt, the acceleration was set as the highest value in the range, at $1.47 \mathrm{~m} / \mathrm{s}^{2}$. The 40 -seat SAV bus would have the lowest acceleration in the range, at $0.9 \mathrm{~m} / \mathrm{s}^{2}$. The other types of vehicles are assumed to have rates in between the small AV and the SAV bus with linear interpolation. Further, the emergency deceleration rate is assumed to align with the normal bus configurations to ensure the comfort of the riders, based on the default SUMO value. Length, width, height, and maximum speed were obtained from the existing sedan, van, and bus size parameters $[26,30,31]$. Compared to conventional vehicles, SAVs are capable of maintaining a closer distance with other vehicles while driving, and connected technology may lead to shorter following distances [36]. Smaller SAVs are nimbler than large-size SAVs, thereby using the road space more efficiently. Adopted from the range that was used in Morando et al.'s [3] simulation, 2 meters is used for the 40-seat SAV bus and 0.5 meters for the 5-seat SAV, with interpolated numbers for 20 -seat and 10 -seat SAVs.

A model from Jara-Díaz and Tirachini [34] showed that the average boarding and alighting time is 3.3 seconds/ passenger using a contactless card or 4 seconds/passenger using the magnetic strip when only the front door is used for boarding and both doors are used for alighting. The current tested automated shuttles have one door for both boarding and alighting, but the door is wider than the mid-sized bus. Here, 3.5 seconds (considering 0.5 -second simulation timestep) is used for the average boarding and alighting time, although there could be variations due to the vehicle design and payment method.
3.4. Scenario Design. This paper tests a base case scenario that has riders' demand varying from 100 riders per hour to 900 riders per hour. Background flow is set to $1260 \mathrm{vphpl}$, without SAV parking bays at the stop or traffic signals. In terms of the travel demand, the simulated average trip distance is 2.37 miles with a standard deviation of 1.40 miles. Based on the demand and capacity, the headway of SAVs is set for each level of demand such that the total demand can be met considering the total available seats of dispatched SAVs, the average assumed load factor of SAVs, and the average trip length, recognizing riders' potential waiting time at the stops. Table 2 gives an example of SAV dispatching headway settings when assuming that SAVs seats are always full (load factor $=1$ ). Assuming one seat can potentially serve 2 person-trips on average along the 6.5-mile corridor, a $2 \mathrm{~min}$ headway of 5-seat SAVs (i.e., $30 \mathrm{SAVs}$ per hour) is required to meet the demand, which equals number of SAVs $\times$ vehicle size $\times$ load factor $\times$ trips $=30 \times 5 \times 1 \times 2=300$ riders per hr. A high load factor indicates a low SAV dispatching frequency. For the base case scenario, a load factor of 0.7 for all types of SAVs is assumed, while a sensitivity analysis of load factor variations is conducted later. Many headway settings here are less than 5 minutes (especially for small-size vehicles), which is uncommonly seen, but this simulation mimics a transit corridor that is often shared by more than one bus route and thus a more frequent service.

Other than the base case scenario, a few scenarios have been designed for comparison, as shown in Table 3. These scenarios include varying the background flow (varying from 0.5 to 0.8 vehicles per second by Poisson distribution), adding station bay and traffic signals, and changing the SAV dispatching headways (via various assumed load factors).

For the station bay scenario, the simulation sets up an extra lane for SAVs to stop only, to mimic the case when there is a parking bay at the station for SAVs. Capacity at the station bay is unlimited (or large enough to hold many SAVs, because of the extra lane), but the SAVs follow the principle of first-come first-leave. For the traffic signal scenario, a 90-second cycle is assumed, with 3 seconds of yellow time and 10 seconds of red time. The signal locations are set every $1 / 8$ miles, which is about 2 signals per station. 
TABLE 2: Example of SAV headway (min) settings for load factor $=1$ assumption.

\begin{tabular}{lcccc}
\hline \multirow{2}{*}{ Demand (SAV users per hour) } & \multicolumn{2}{c}{ Capacity (seats per SAV) } & \\
& 5 seats per SAV & 10 seats & 20 seats & 40 seats \\
\hline 100 people/hr & 6 min headway & 12 min & 48 min \\
200 & 3 & 6 & 12 & 24 \\
300 & 2 & 4 & 8 & 16 \\
\hline
\end{tabular}

TABle 3: Tested scenarios.

\begin{tabular}{|c|c|c|c|c|c|}
\hline \multicolumn{2}{|c|}{ Base case } & Background flow (vphpl) & Load factor & Traffic signal & Station bay \\
\hline \multirow{5}{*}{ Sensitivity analysis } & & 1260 & 0.7 & - & - \\
\hline & Background flow & $900,1080,1260,1440$ & 0.7 & - & - \\
\hline & Station bay & 1260 & 0.7 & - & $\checkmark$ \\
\hline & Traffic signal & 1260 & 0.7 & $\checkmark$ & - \\
\hline & Load factor & 1260 & $0.5,0.6,0.7,0.8$ & - & - \\
\hline
\end{tabular}

Since this transit corridor focuses on the investigations about SAV size and frequency under different travel demands, the locations of traffic lights and the signal timing are arbitrary. Optimization techniques could be involved in a real-world application.

3.5. Evaluation Metrics. The average background vehicle travel speed, average rider waiting time, total vehicle travel time, total rider travel time, and total system cost per traveler will be evaluated for each scenario. The background vehicle travel speed (vehicles) can evaluate how congested the traffic is, while the average person waiting time shows the efficiency of the transit system. The total travel times of background vehicles and SAVs can show the overall system performance, and the total system cost per traveler will evaluate the cost to serve travel demand under different types of vehicles and levels of service. The total system cost per traveler considers the travel time cost and operating cost of SAVs and background vehicles. Table 4 shows the detailed components of the total system cost. When riding in an SAV, riders are assumed to perceive a value of travel time (VOTT) that is half that of those human-driven vehicles. However, when they are walking to and from SAV stop locations or waiting for an SAV to arrive, their VOTT is assumed to be double that of a driver [37]. The vehicle cost is calculated using a per-vehicle mile basis. The per-mile cost is adapted from Bösch et al. [5], which considered both fixed cost and variable cost for different sizes of SAVs on a per vehicle-mile basis. The total system cost is further transformed to cost per traveler, by assuming an average occupancy of 1.2 in background vehicles.

\section{Results}

4.1. Base Case, SAV Station Bay, and Traffic Signal Scenarios. In this section, the analyses of three scenarios are presented: the base case scenario, adding SAV station bays, and adding traffic signals. Each scenario performed five runs and the average value is shown in the results. Figure 3 shows the results of the base case scenario. About 7,450 personal vehicles pass through the transit corridor during the 3 hour simulation time, with the number of dispatched SAVs varying from 5 to 386, depending on the demand level and SAV size.

Figure 3(a) presents the relationship between different levels of demand and the average background vehicle travel speed for all vehicle sizes. At a very low demand level (i.e., 100 people/hr), vehicle size hardly affects the average speed of background vehicles, because vehicles stop less frequently. However, the speed of the average background vehicles decreases with increasing passenger demand for all demand levels. More frequent stops are witnessed at higher demand levels, to accommodate greater passenger demand, thus affecting the background traffic. 40-seat SAVs are outperformed by other sizes of SAVs because these large-size vehicles accelerate and decelerate slower and are more likely to dwell frequently at stops to serve riders, while small-size vehicles are more likely to skip stops due to full capacity. Interestingly, the downward trend of decreasing background flow speed in the 5-seat SAV scenario is more prominent than SAVs of other sizes, and the background vehicle speed is lower than 10-seat SAVs when demand is greater than 500 people/hr and further lower than 20-seat SAVs when demand is greater than 900 people/hr. This is due to more dispatched SAVs in the corridor which causes congestion from SAVs driving instead of dwelling at the stops.

Figure 3(b) shows an expected result: SAVs with more frequent service reduce people/passenger waiting times at all passenger demand levels. Average waiting time discrepancies between different SAV sizes shrink under a higher demand level due to a higher frequency of all types of vehicles and a large demand base. However, the scale does not change. For example, 40-seat SAVs result in an average passenger wait time 3 times longer than 5-seat SAVs across all demand levels.

Total vehicle travel time and total rider travel time (walk + wait + ride) are shown in Figures 3(c) and 3(d), respectively. Total vehicle travel time increases with the travel demand (Figure 3(c)), especially for 5-seat SAVs, mainly because there are more vehicles dispatched into the corridor to serve riders that further obstruct more background vehicles for a longer time. High demand levels witness a clear trend, where the increasing trend of 5-seat 
TABLE 4: Components of total system cost (drivers' VOTT $=\$ 15 / h r$ ).

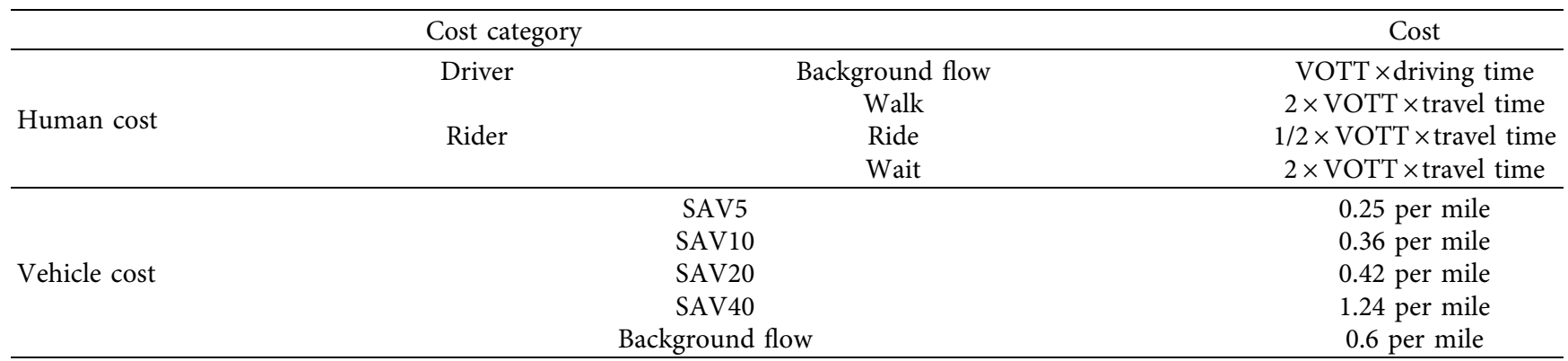

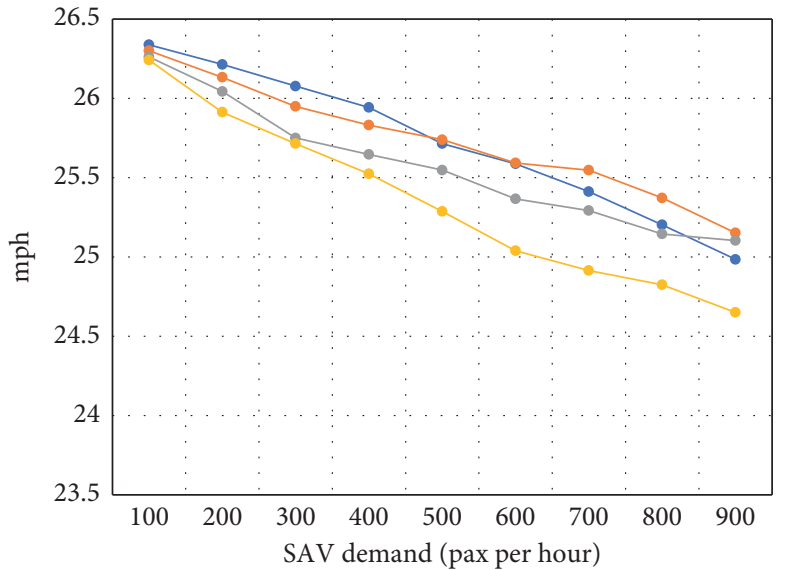

$\rightarrow$ 5-seat SAV

$\rightarrow$ 20-seat SAV

$\because 10$-seat SAV

(a)

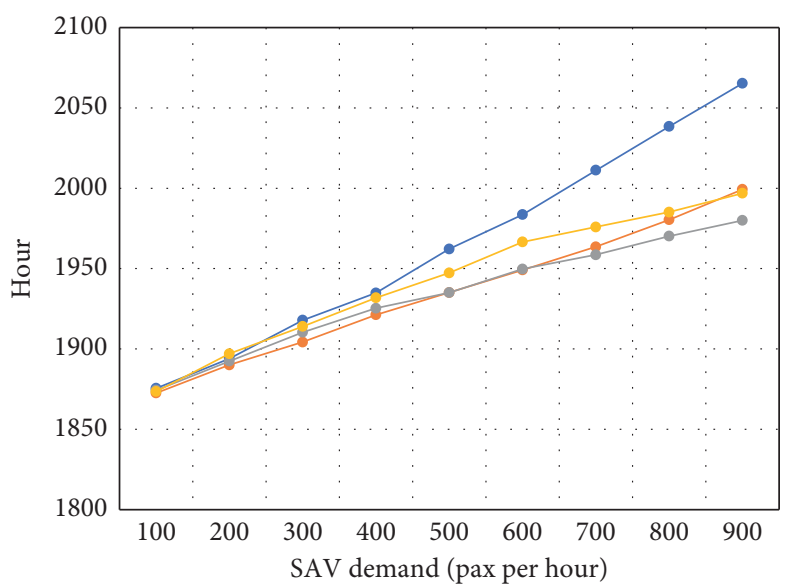

$\longrightarrow$ 5-seat SAV

$\rightarrow 10$-seat SAY

$\rightarrow$ 20-seat SAV

$\rightarrow$ 40-seat SAV

(c)

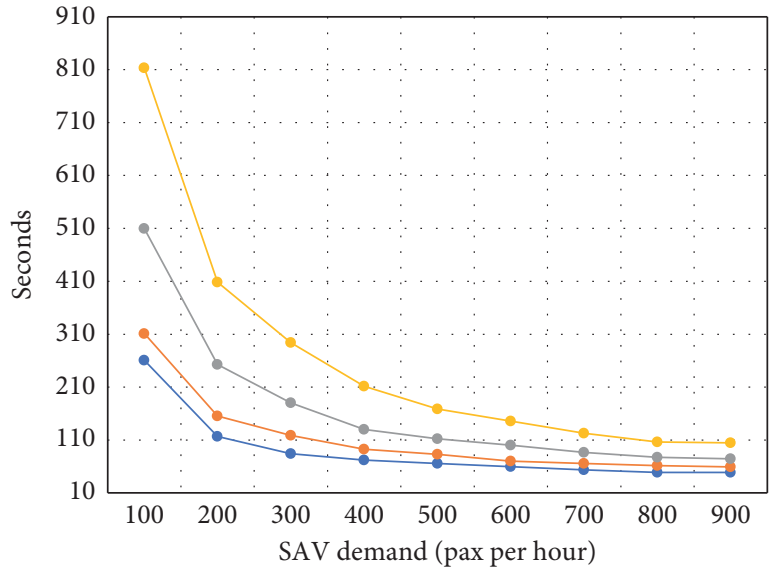

$\longrightarrow$ 5-seat SAV

$\rightarrow 10$-seat SAV

$\longrightarrow 20$-seat SAV

$\because 40$-seat SAV

(b)

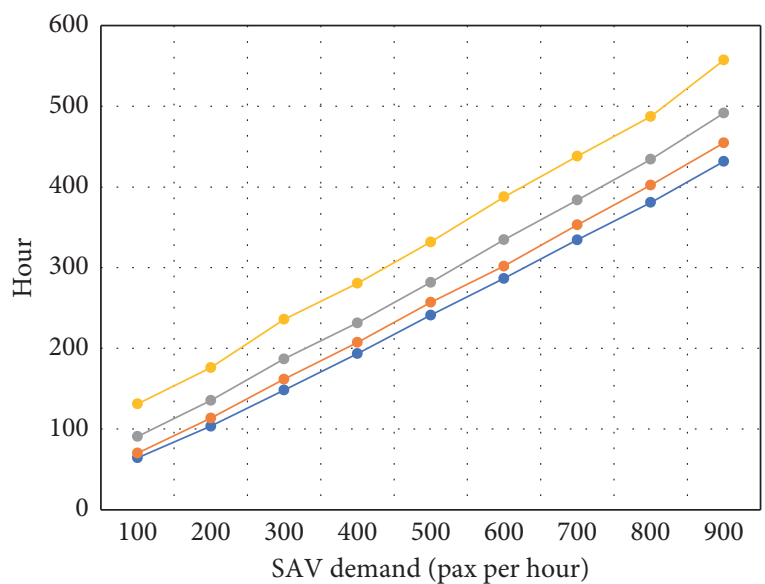

$\begin{array}{ll}\rightarrow \text { 5-seat SAV } & \rightarrow \text { 20-seat SAV } \\ \longrightarrow \text { 10-seat SAV } & \rightarrow \text { 40-seat SAV }\end{array}$

(d)

Figure 3: Continued. 


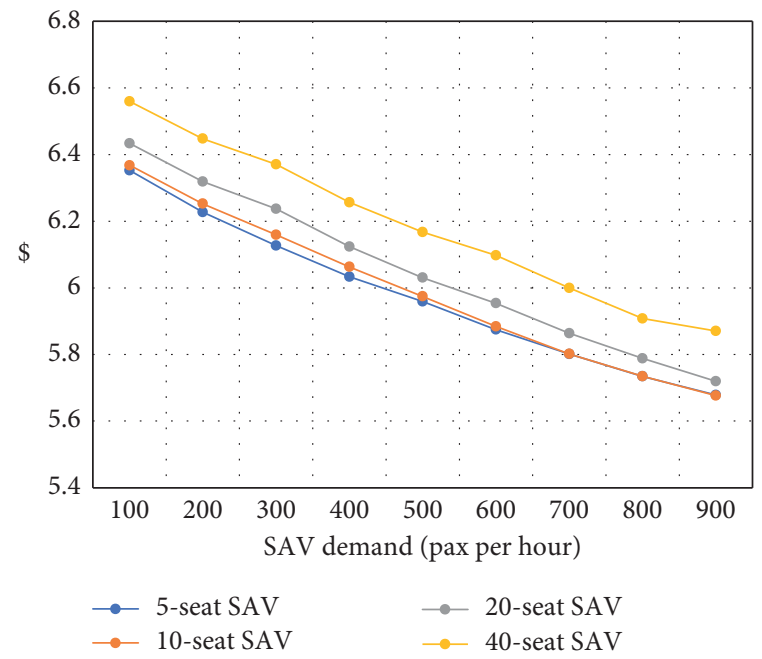

(e)

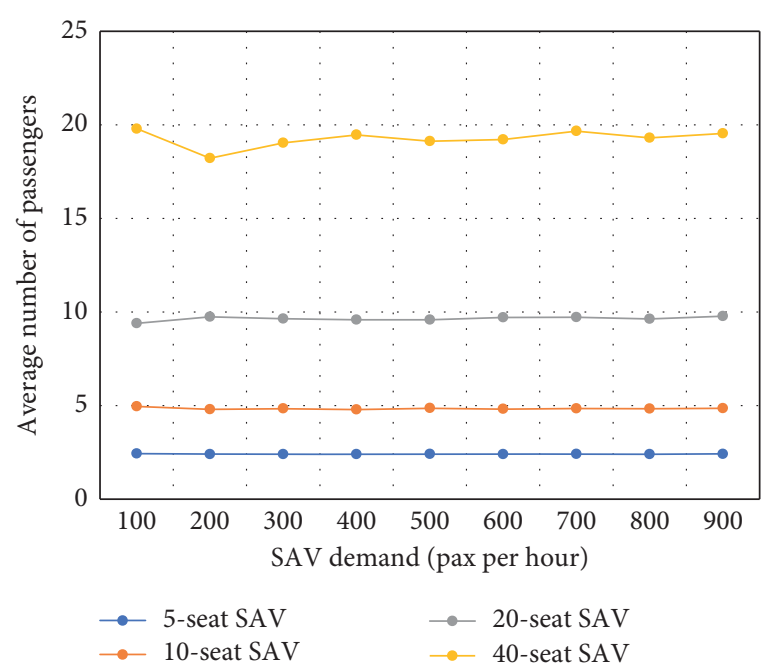

(f)

Figure 3: Base case scenario. (a) Background vehicle average speed. (b) Average wait time per SAV rider. (c) Total vehicle travel time. (d) Total rider travel time. (e) Total system cost per traveler. (f) Average vehicle occupancy.

SAVs and 10-seat SAVs keeps going up, but the trend of 20seat SAVs and 40-seat SAVs tends to flatten out. Of course, more rider demand also leads to more total rider time (Figure 3(d)), but small-size SAVs (with small headways) tend to decrease wait time, and thus their riders experience less journey time across all demand levels.

While using smaller SAVs reduces passenger waiting time, it can increase total vehicle travel time and riders' total journey time at high demand levels. Regarding this, the smallest size of SAVs is not necessarily the preferred vehicle size, and thus the total system cost per traveler is evaluated. Figure 3(e) illustrates the result of total system cost per traveler where all sizes of SAVs enjoy the scale of economy, as seen from the decreasing trend. The 40-seat SAVs and 20seat SAVs are outperformed by smaller SAVs at all demand levels, respectively. Compared to the 40-seat SAVs, using smaller SAVs would reduce the system cost by up to $4.0 \%$. While the 5-seat SAVs have lower total costs with a demand of 700 people/hr or less, the 10-seat SAVs have lower total costs with a demand of 700 people/hr or more. The trend indicates that there is a chance for 20-seat SAVs to be the optimal vehicle size at a higher demand level, but one would not expect it to happen in many middle- or large-size cities.

To differentiate the revealed load factor (or simulated load factor) of the SAVs, the ratio of person-miles to seatmiles is expressed as the average vehicle occupancy. Figure 3(f) shows such metrics for each vehicle type, which turns out to be stable (about half of vehicle space filled up on average) across vehicle sizes. The reason could be the assumption of SAV dispatching frequency, which is a fixed relationship with vehicle size and passenger demand, as illustrated in Table 2. The SAV occupancy of different vehicle sizes is about 50\%, which is lower than the targeted load factor 0.7 , due to fewer alighting at the beginning of the corridor and fewer boardings at the end of the corridor. With less dispatch frequency, the average vehicle occupancy is likely to increase, but the riders will wait longer at the stops. This will be further analyzed in sensitivity tests.

Figure 4 summarizes the results of the SAV station bay scenario, showing the new patterns of the average speed of background vehicles and the total cost per traveler, as well as their responding changes from the base case scenario. As the stops are now in bays, the impact of SAVs on background traffic becomes negligible, especially for large-size SAVs, as shown in Figure 4(a). This result aligns with the real-world cases of human-driven vehicles, e.g., Koshy and Arasan [38] and Zhang et al. [39]. Although there is little difference in average travel speed for various demand levels, 5-seat SAVs still show a decreasing trend for a large demand due to frequent bay entries or exits. Compared with the base case scenario (Figure 4(b)), bus-bay provision has more benefits for large-size SAVs across all demand levels, making them competitive to small vehicles in terms of the corridor's average speed. However, 40-seat SAVs are still outperformed by smaller sizes of SAVs, for all demand situations, due to the high cost of large-size SAV operations. Both 5-seat and 10-seat SAVs tend to be more cost-efficient under different levels of travel demand. Using smaller-size SAVs instead of 40 -seat SAVs can reduce the system cost by up to $3.2 \%$, which is consistent with the base case scenario, although the benefit shrinks by $0.8 \%$ due to the use of bays. This is because the bays mitigate the situation where SAVs previously obstructed the following vehicles and such benefits become more prominent when the riding demand is large (i.e., more stops at the stations). This can also be observed from the total system cost saving per traveler (Figure 4(d)), where the small-size SAVs deliver slightly more benefits across all demand levels, but more benefits are delivered under cases of large demand and large-size SAVs. It is worth noting that the bus-bay provision also adds construction costs and potentially right-of-way acquisition costs. For actual applications, site-specific details and a more comprehensive cost-benefit 


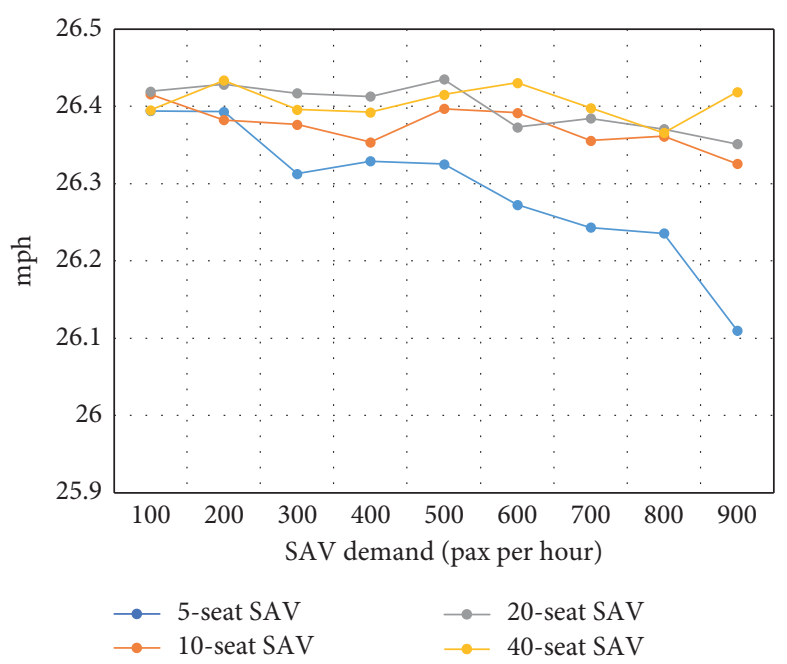

(a)

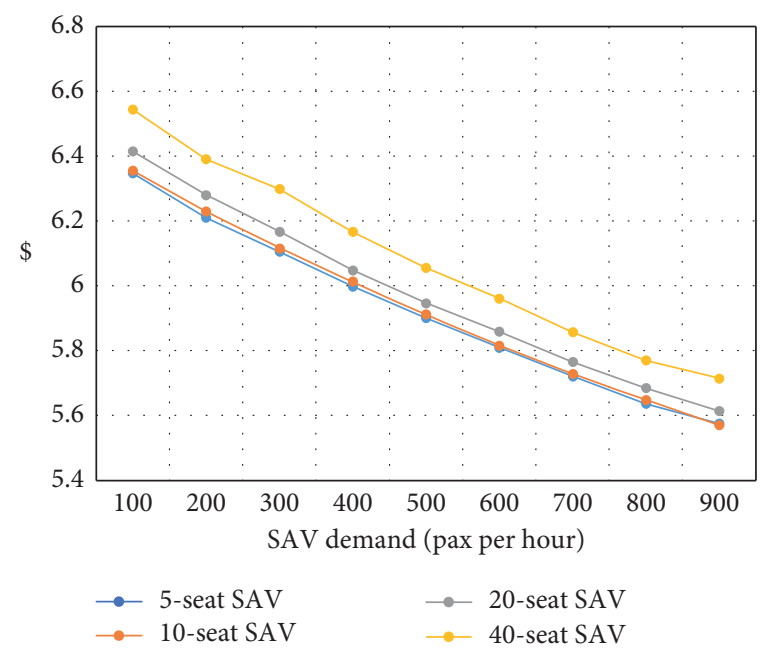

(c)

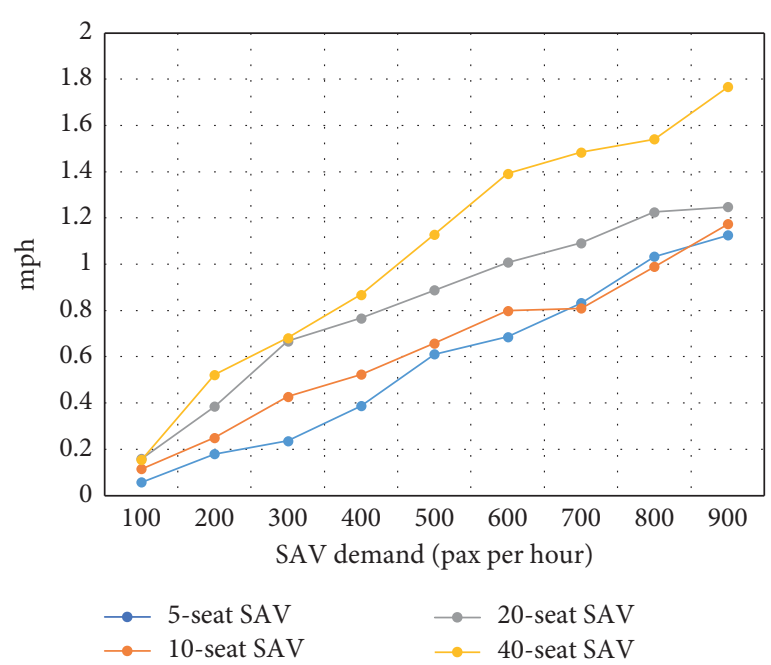

(b)

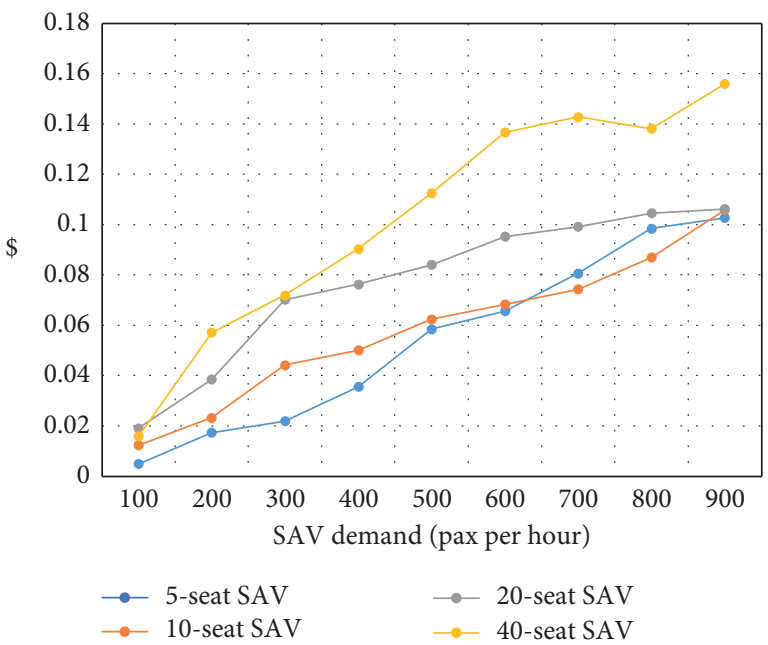

(d)

FIgURE 4: SAV station with the bay scenario. (a) Background vehicle average speed. (b) Increase of background vehicle average speed (compared to the base case). (c) Total system cost per traveler. (d) Total system cost saving per traveler (compared to the base case).

analysis will be needed, before sizing and scheduling SAV transit, recognizing factors like more cross-traffic and/or pedestrians at certain intersections, higher SAV ridership-with longer boarding times and oncoming traffic.

Results of the traffic signal scenario are presented in Figure 5. Figures 5(a) and 5(b) show that the average speed of background vehicles is substantially lower compared to the base case scenario due to the delay at traffic signals, dropping by about $3.5 \mathrm{miles} / \mathrm{hr}$ on average. This speed reduction may vary based on intersection type, signal phases, and average speed, as shown in existing literature [40, 41]. Due to congestion caused by more 5-seat SAVs dispatched to the corridor and thus more delays caused by the traffic signals, the average background vehicle speed for the 5-seat SAV scenario is lower than the 40-seat SAV scenario when demand is greater than 800 people/hr.

The heavy external cost by traffic signals for 5-seat vehicles can also be observed in Figure 3(d), where the total system cost per traveler is greater than that of 20-seat SAVs and starts to flatten out when the demand is greater than 800 people/hr. Such impacts can also be observed in Figure 5(d), as the 5-seat SAVs do not enjoy the scale of economy at high demand, compared to the base case. The general downward trend of total system cost per traveler for SAV sizes remains similar, and the 40-seat SAVs still have the highest cost across all demand levels. The best vehicle choice, however, is different from the base case scenario. 5-seat SAVs are only preferred when demand is lower than 300 people/hr, while the 10-seat SAVs are favored when demand is greater.

4.2. Sensitivity Tests on Service Frequency and Background Flow. Figure 6 demonstrates the effects of SAV headways (via changing assumed load factors) on the base case scenario, by showing changes in background vehicle average speed, total system cost per traveler, and average wait time per SAV rider. The average speed of the corridor remains relatively stable across travel demand levels when varying 


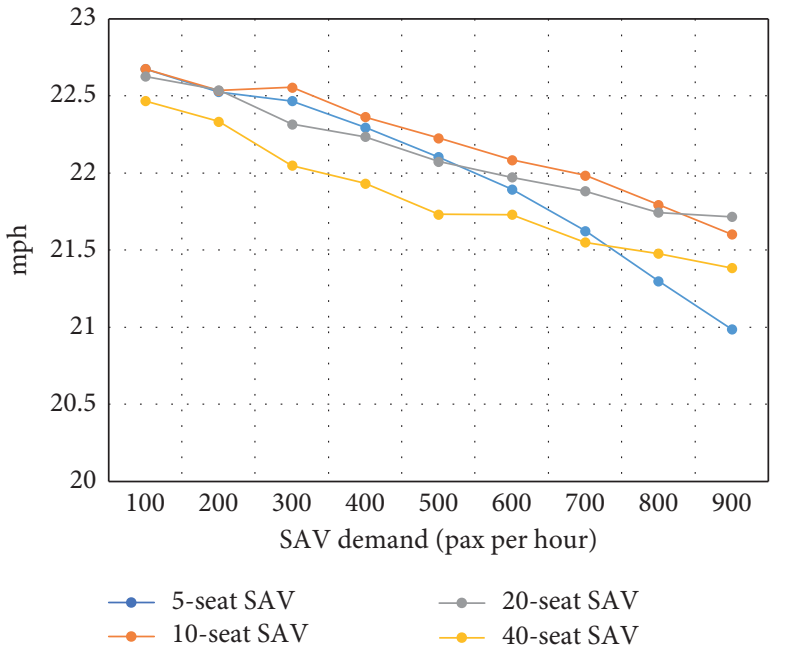

(a)

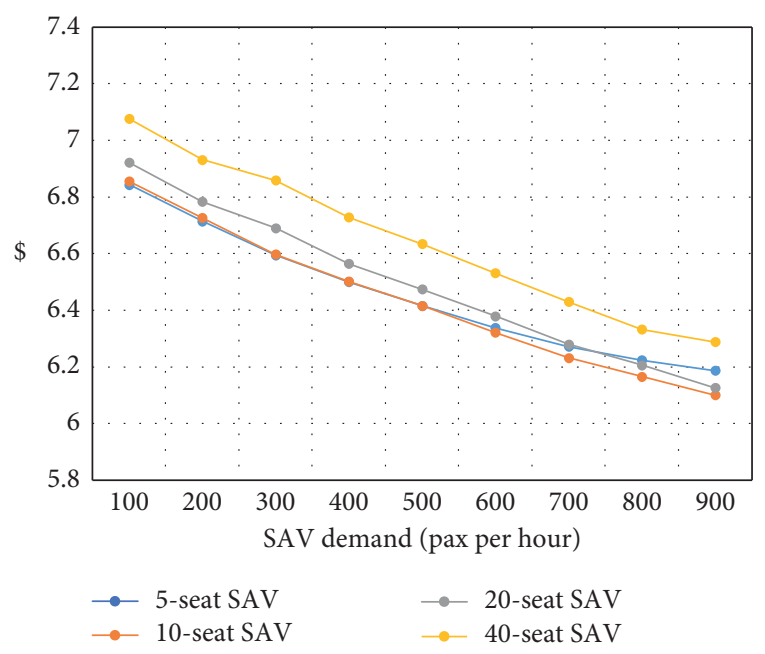

(c)

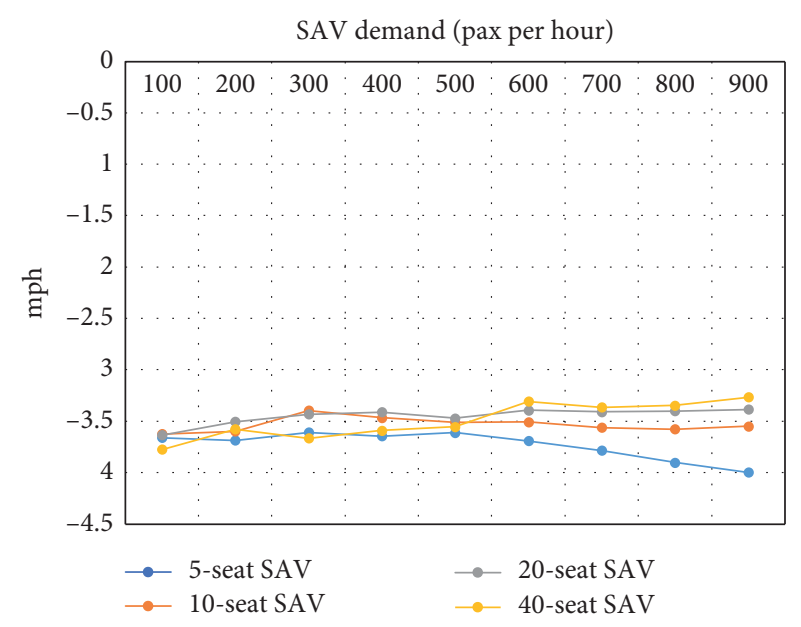

(b)

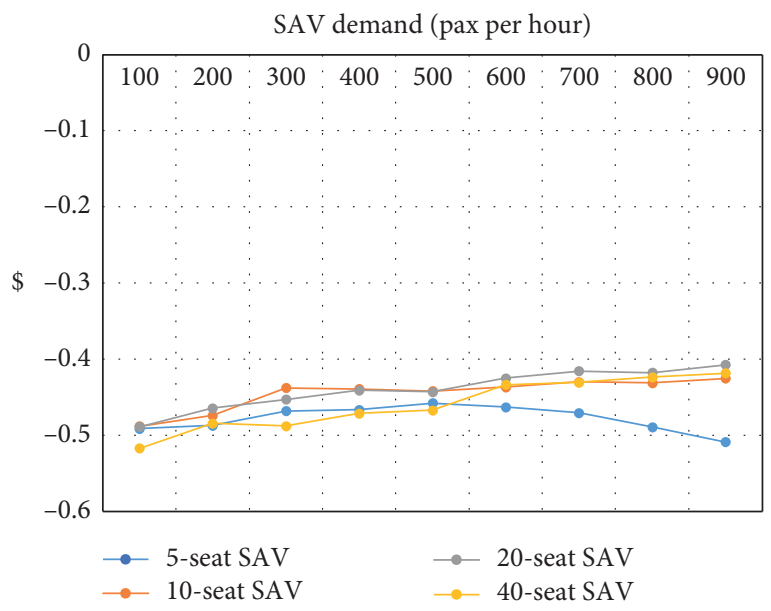

(d)

Figure 5: Traffic signal scenario. (a) Background vehicle average speed. (b) Increase of background vehicle average speed (compared to the base case). (c) Total system cost per traveler. (d) Total system cost saving per traveler (compared to the base case).
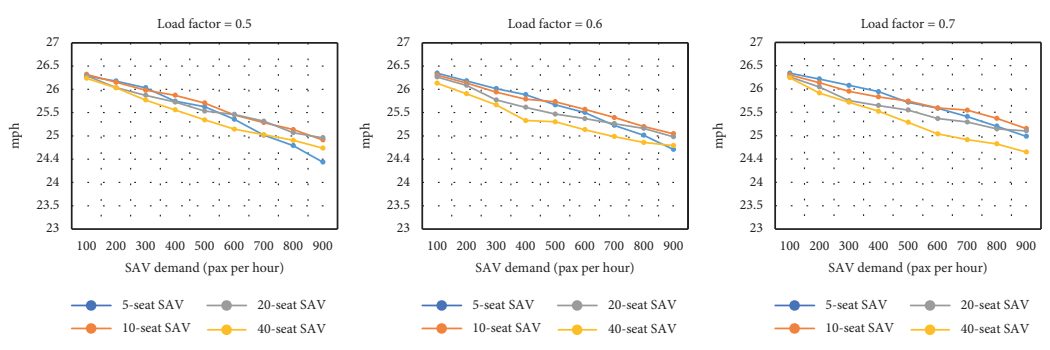

(a)
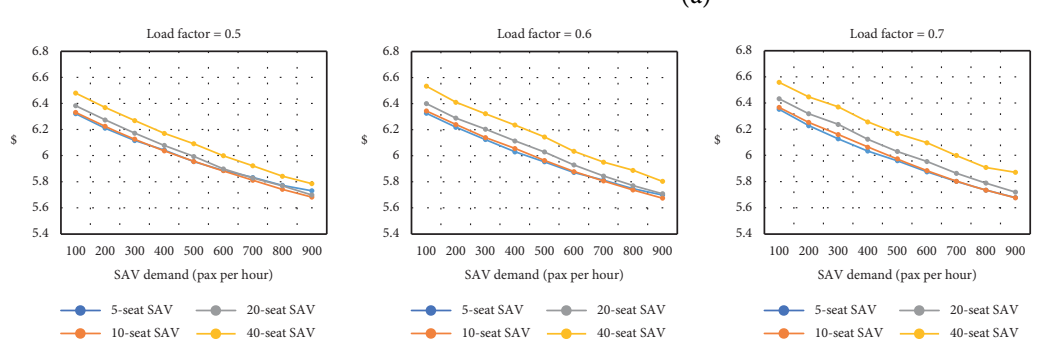
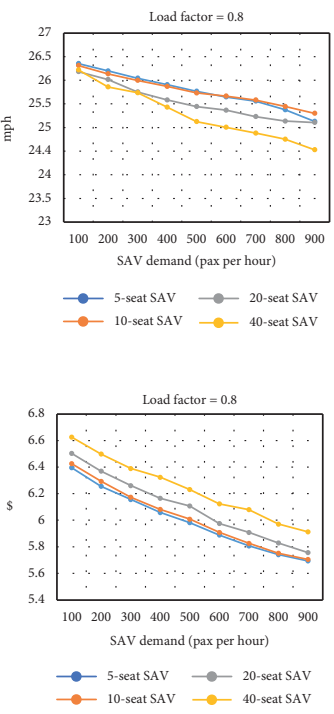

(b)

Figure 6: Continued. 

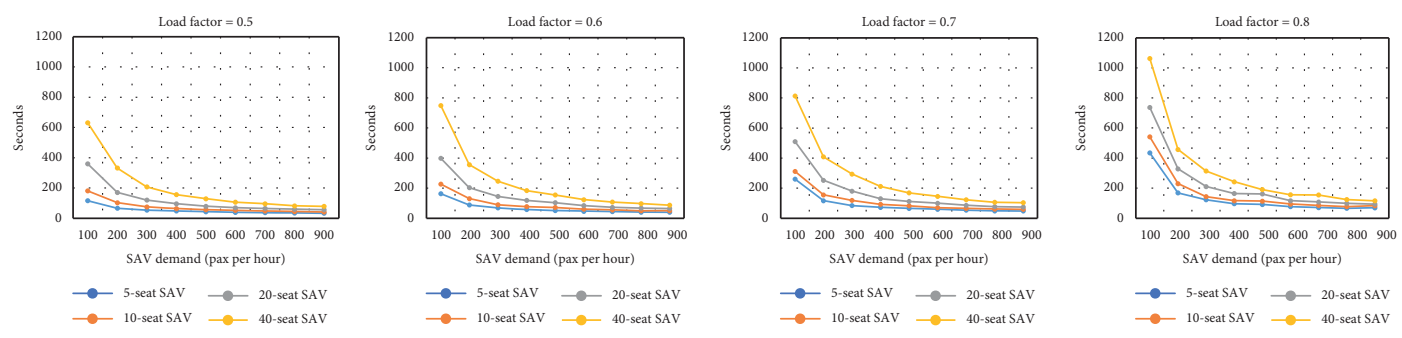

(c)

FIgURE 6: Sensitivity analysis on varying load factors (SAVs' dispatching frequency). (a) Background vehicle average speed (vphpl). (b) Total system cost per traveler (\$). (c) Average wait time per SAV rider (second).

SAV dispatching frequency, although the preferred size of vehicle changes. Small-size SAVs offer faster speeds when the vehicles are dispatched less frequently, due to less congestion brought by fewer vehicles and fewer obstructions to background vehicles when dwelling at the stops. Although a more frequent service will lead to higher vehicle operating costs, the total system cost per traveler decreases slightly for each vehicle size, due to less wait time at the stops for riders (Figure 6(b)). 5-seat SAVs are cost-efficient across all demand levels when the SAVs are dispatched infrequently (assuming load factor $=0.8$ ), while 10-seat SAVs are favored at high demand (greater than 400 people/hr) when service is frequent (assuming load factor $=0.5$ ). However, 40-seat SAVs are still outperformed at all demand levels in terms of the total system cost per traveler. The average vehicle occupancy of the SAVs is stable when there are varying vehicle sizes but increases with less frequent service, from loading $34 \%$ (assuming load factor $=0.5$ ) to $55 \%$ (assuming load factor $=0.8$ ).

Different background flow rates were also tested for the base case scenario. Figure 7 shows the background vehicle travel speed and total system cost with background flow rates of 900, 1080, 1260 (base case scenario), and $1440 \mathrm{vphpl}$. Of course, a high rate of background flow would lead to a more congested corridor. When increasing the background flow from $900 \mathrm{vphpl}$ to $1440 \mathrm{vphpl}$, average background flow speed for the 5-seat SAV scenario drops by up to $8.0 \%$ (at high demand level $=900$ people $/ \mathrm{hr}$ ) and average background flow speed for the 40 -seat SAV scenario drops by up to $11.4 \%$ (at high demand level $=900$ people/hr). When the background flow is low, the corridor enjoys a higher speed provided by large-size vehicles, because there are few of them to obstruct the background flow. For example, 40-seat SAVs obstruct background flows the least, at $900 \mathrm{vphpl}$, across all demand levels. However, the nimble small-size SAVs show benefits as the corridor becomes congested. At the background flow rate of $1440 \mathrm{vphpl}, 5$-seat SAVs provide the least obstruction under passenger demand less than 500 people/hr and 10-seat SAVs enable faster speeds for higher demand levels, while 40-seat SAVs lead to the lowest speed across all levels of demand. In terms of the total system cost per traveler by different levels of demand, the decreasing trend of cost per traveler in sensitivity scenarios follows that of the base case scenario with 40 -seat SAVs still being the least cost-efficient. Generally, 5-seat and 10-seat SAVs perform relatively well at total system cost per traveler, with 5-seat SAVs performing slightly better at lower demand levels and 10-seat SAVs better at high demand levels. Of course, the congestion caused by a higher rate of background flow would lead to an increase in the total system cost per traveler. Such an increment for all sizes of SAVs is small at low travel demand levels (about a 3.5\% increase) compared to that at high travel demand levels (about a 9.5\% increase). These results suggest that large-size SAVs provide slightly better corridor average speed under low background traffic, but small-size SAVs (e.g., 5-seat and 10-seat SAVs) would be more cost-efficient at all congestion and demand levels.

\section{Discussion}

5.1. From Human-Driven to Automation. At present, conventional vans have been used to provide mobility in confined regions like college campuses, military bases, airports, or business parks, where the SAVs are more likely to be in the early stages of implementation. Of course, SAVs can provide safety and cost benefits compared to vans, as SAVs have better control while eliminating the need to hire a driver. However, the benefits of SAVs are also expected in the case of serving a busy transit corridor, where 40 -seat conventional buses are still dominating currently. This is because 40 -seat buses, which require a smaller fleet size compared to using small-size vehicles, allow the fleet operator to efficiently (both in time and cost) manage the drivers (along with their schedule) and the bus fleet, in terms of routing and parking. As an emerging technology, SAVs provide the opportunity of a flexible design of vehicle size as shown in various global tests that are currently being conducted [19], while the fleet manager does not have to worry about the cost and time burden of driver and vehicle assignment and relocation. One driver is usually assigned to one or two bus lines he/she is familiar with, but SAVs are capable and flexible when switching to other lines. In order to perform complex interactions with humans and other vehicles, such an SAV service would require a mature automation technology with a high initial cost, but the cost savings they bring would be substantial due to their efficient routing and repositioning without drivers. Although conventional bus transit scenarios, usually with larger vehicles, are not simulated in this study, their system costs and passenger waiting times would be higher than the 40 -seat 

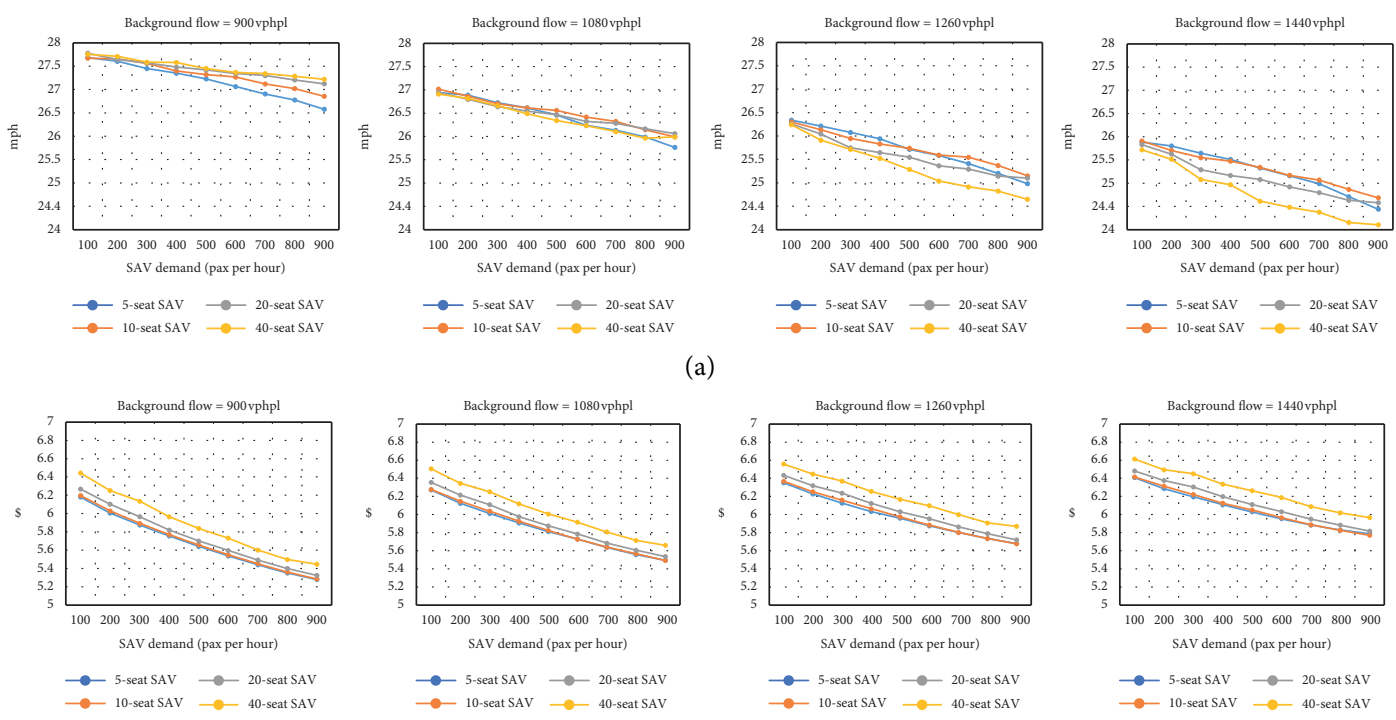

(a)
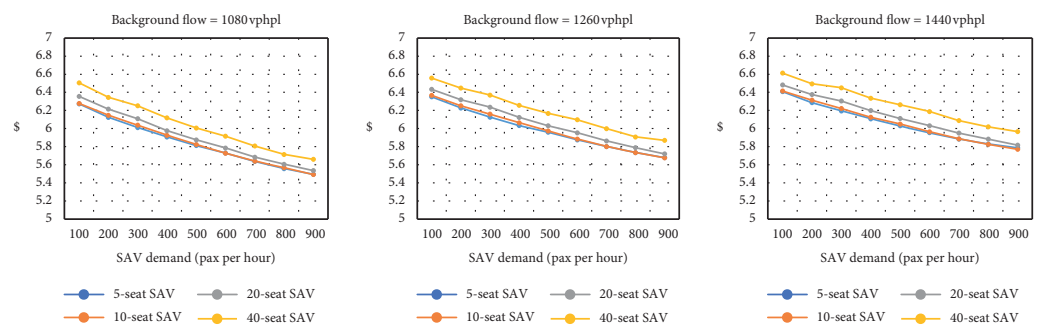

(b)

FiguRE 7: Sensitivity analysis on varying background vehicle flow rates. (a) Background vehicle average speed (vphpl). (b) Total system cost per traveler $(\$)$.

SAV scenarios, due to SAVs' reduced driver cost, increased safety, and less emission from smooth acceleration and deceleration. Thus, replacing conventional bus transit vehicles with SAVs of smaller sizes would offer greater reductions in costs.

5.2. Insights for Real-World Applications. Different scenarios have been tested on the impacts of demand, vehicle size, traffic signals, station bays, load factor, and background vehicle flow rate, but results may still change in practice due to the other assumptions made about the corridor. Changing the speed limit may not change the pattern of preferred SAV size, but riders' cost per PMT will increase or decrease with the speed limit change, due to the travel time change traversing the whole corridor. However, a higher speed limit means that SAVs may need a longer stopping distance, or brake early, leading to longer queues at the stops and thus causing more delay along the corridor. The spacing between stops may be adjusted in the real world, based on different land-use patterns. Moreover, a stop location can be a far-side stop (after buses proceeding through the intersection), nearside stop (before the intersection), or midblock stop (in between intersections, usually in a well-defined area), and choices should also be adjusted based on the road geometry and the real traffic volume, to minimize conflicts with turning vehicles and reduce intersection clearance time [42].

Although the bus station bay and traffic signal scenarios have been tested, the real impacts of background vehicles entering and exiting the corridors are not considered. The background vehicle in the real world may not enter and exit at either end of the corridor, but will enter or exit at any point along the corridor instead. This causes more delay in the corridor, the impact of which may even be worse than SAVs dwelling at the stops. Such delay cost from background vehicles as well as their potential parking cost may largely increase the real system cost along the corridor. A different arrival rate of passenger demand may be witnessed in the real world, instead of the passengers being uniformly distributed along the corridor. The arrival rate may change based on different times of day, as the system will serve more travel demand during the morning and afternoon peak hours. Land-use patterns will also impact the passengers' arrival distribution at the stops, as a stop near a commercial area or a transfer stop may attract more demand. Change in land-use pattern is more outstanding when considering a corridor that is 6.5 miles long, which is likely to go through the urban core and end at a suburban area in some small-size cities. More boardings and alightings will be observed at the urban core, leading to more delays at the SAV stops, especially during peak hours when the corridor may already be congested. On the contrary, the suburban area will bear less travel demand and the vehicle will be less utilized (i.e., low AVO). Moreover, a real-world transit corridor can be longer than 6.5 miles with different transit lines often sharing the same corridor segments. This brings more variations to the analysis due to more complex interactions among the transit vehicles and passengers, such as transfers between transit or other multimodal travel methods (e.g., park-and-ride or bike-and-ride).

5.3. Planning and Policy Implications. Simulation results illustrate the total-cost benefits of smaller SAVs (i.e., 5-seat or 10-seat SAVs), to serve as public transit vehicles along existing lines or in new locations. Such high-frequency, smaller-vehicle transit-type systems can better serve travel demands in many markets while reducing the heavy footprint of motorized modes (by encouraging more shared rides). Travel demand from both existing transit users and the new users who enjoy the comfort and the low cost of the new SAV transit service must be considered when planning 
new SAV transit lines. Signals should be involved in the decision of the service frequency, since the use of signals decreases the corridor's average speed and causes more delays to the whole corridor in addition to the delays caused by SAVs dwelling at stops. Bays can mitigate the negative effect of SAVs blocking one lane when dwelling and can be more effective at minimizing such an effect under the existence of traffic signals. However, since acquiring the right of way is usually expensive, bus bays should be constructed wisely at different locations of the corridor.

Based on a variety of designed SAV sizes, there is a possibility that smaller SAVs can expand transit service areas to where large-size bus lines could not previously reach (due to low AVO, and thus poor profits). SAVs can run longer transit lines, overlapping existing line segments that largesize buses use where the demand is high. This could function similarly to current subway systems across the globe, where an extra fleet will provide more frequent service at the urban core area of a transit line [43]. SAVs can help reduce the long access and egress times of many riders, especially those who live near the ends of the lines. Moreover, smaller SAVs may also provide an option for riders who enjoy a more comfortable space (i.e., 4-seat SAV) to rest or work, rather than sharing a bus with 30 or more riders. A higher fare may be priced for riders who want to take small SAVs, but fares can be low when services are first introduced to incentivize riders to use such public transit service.

With the benefit of eliminating driver costs and SAVs' ability to self-relocate, the transit operators may own a larger fleet, due to the use of new transit lines and potential service with mixed-size SAV fleets. A larger parking depot may be desired for such a large fleet, but the SAVs are also expected to look for proper social parking sites by themselves so that they can utilize vacant overnight parking spaces. As the world is migrating to vehicle electrification, shared automated electric vehicles (SAEVs) are also expected to drive themselves to charging stations throughout the day, by navigating to available stations. It is also worth noting that as the case of fixed-route SAV service is investigated in this paper, the demand is still expected to be relatively high and dense around the stops. When the demand pattern is sparse, the on-demand service is potentially more efficient [44].

5.4. Limitations. Some other limitations of the microsimulation in this study also exist. The relationship between headway and demand is assumed to be fixed, based on the assumed load factor of SAVs. Optimization techniques could be utilized to find the best headway as well as vehicle size for the most cost-efficient system, but these techniques would not be easy to integrate. On the other hand, considering the complex behavior of buses waiting for approaching riders and skipping stops when the seats are full is much easier to integrate. Furthermore, it remains uncertain whether future 40seat SAVs would be able to provide standing area, in which case the capacity of the vehicle would be more than 40 riders. This study assumes that the capacity of a 40 -seat SAV is 40 riders, but 40 -seat SAVs have the potential to be favored by operators if standing is allowed on board. Moreover, the results are only averaged over five random runs, due to the computation burden. The trends are generally clear, but increasing the number of runs can help reduce the variations in the data points.

\section{Conclusion}

This study investigated the performance of an SAV-based bus transit corridor, where different sizes of SAVs replace conventional bus transit vehicles. SUMO was used to simulate microscopic interactions between SAVs and background traffic, and between SAVs and transit passengers under various background traffic conditions, SAV sizes and associated characteristics, passenger demand levels, and load factors. Different configurations of the 6.5-mile bus transit corridor were considered, including a nonsignalized corridor, signalized corridor, and corridor with SAV stops in bays. Detailed bus behaviors were incorporated, including waiting for approaching riders and skipping stops when the vehicle capacity is reached.

Simulation results show that the use of smaller, but more frequent, SAVs leads to reductions in passenger waiting times but increases in total vehicle travel times. It is found that more frequent services of smaller SAVs do not, in general, significantly affect background traffic given their shorter dwell times at stops, as seen from the fastest speed provided by 5 -seat SAVs for passenger demand under 400 people/hr, and by 10-seat SAVs for demand higher than 400 people/hr in the base case scenario.

Results highlight that the systems with 5-seat or 10-seat SAVs have lower total system cost per traveler than those with 20-seat or 40-seat SAVs, consistently across most scenarios. While the system with 5-seat SAVs has relatively low total system cost per traveler at low passenger demand levels, 10-seat SAVs are favored at higher demand levels. Whether 5-seat or 10-seat, SAVs are the most cost-efficient for a certain level of demand based on the background flow rate and how frequently the SAVs are dispatched to the corridor. Higher background flow and more frequent service would make 10-seat SAVs more appealing, mainly due to efficient vehicle space and fewer vehicles obstructing the traffic compared to 5-seat SAVs. However, 40-seat SAVs are outperformed in total system cost per traveler by other vehicle sizes across all scenarios. Compared to the 40-seat SAVs, using smaller SAVs would reduce the system cost per traveler by up to $4.0 \%$, while improving transit passenger experience with reduced waiting times.

Bus-bay provision helps large-size vehicles offer a higher corridor speed, due to fewer vehicles dispatched in the corridor, but the high operating cost allows them to be outperformed by smaller sizes of SAVs in terms of total system cost per traveler. The use of traffic signals substantially lowers the corridor speed, especially for 5-seat vehicles at high passenger demand, making 10-seat SAVs the best choice for most of the demand levels. Results also suggest that the smallest SAVs are not always the optimum solutions; right-sized SAVs and associated frequencies should be considered based on passenger demand, network configuration, and loading factors. 
It should be acknowledged that, in the microsimulation, background traffic is simulated with typical driving behaviors. Future research could explore the impacts of SAVbased transit when background traffic is also partly or fully automated by considering different AV penetration rates. For a high-frequency bus corridor, platooning of SAV-based transit vehicles should also be considered, particularly when the flow of small-size SAVs would be high. Future work may also examine the impacts of SAVs' vehicle size on the system's energy consumption.

\section{Data Availability}

The data used to support the findings of this study are available from the corresponding author upon request.

\section{Disclosure}

This study has been presented both at the Bridging Transportation Researchers Conference (August 2020) and in 100th Annual Meeting of the Transportation Research Board (January 2021).

\section{Conflicts of Interest}

The authors declare that they have no conflicts of interest.

\section{Acknowledgments}

The authors thank Jade (Maizy) Jeong for her editing and administrative support.

\section{References}

[1] D. J. Fagnant and K. Kockelman, "Preparing a nation for autonomous vehicles: opportunities, barriers and policy recommendations," Transportation Research Part A: Policy and Practice, vol. 77, pp. 167-181, 2015.

[2] J. B. Greenblatt and S. Shaheen, "Automated vehicles, ondemand mobility, and environmental impacts," Current Sustainable/renewable Energy Reports, vol. 2, no. 3, pp. 74-81, 2015.

[3] M. M. Morando, Q. Tian, L. T. Truong, and H. L. Vu, "Studying the safety impact of autonomous vehicles using simulation-based surrogate safety measures," Journal of Advanced Transportation, vol. 2018, Article ID 6135183, 2018.

[4] D. Tian, J. Zhou, Y. Wang, Z. Sheng, H. Xia, and Z. Yi, "Modeling chain collisions in vehicular networks with variable penetration rates," Transportation Research Part C: Emerging Technologies, vol. 69, pp. 36-59, 2016.

[5] P. M. Bösch, F. Becker, H. Becker, and K. W. Axhausen, "Cost-based analysis of autonomous mobility services," Transport Policy, vol. 64, pp. 76-91, 2018.

[6] L. Zhao and A. A. Malikopoulos, "Enhanced mobility with connectivity and automation: a review of shared autonomous vehicle systems," 2019, http://arxiv.org/abs/1905.12602.

[7] CB Insight, 40+ Corporations Working on Autonomous Vehicles, CB Insights, New York, NY, USA, 2019, https://www. cbinsights.com/research/autonomous-driverless-vehiclescorporations-list/.
[8] Waymo, Say Hello to Waymo, Waymo, Mountain View, CA, USA, 2017, https://orfe.princeton.edu/ alaink/SmartDriving Cars/PDFs/AHB30_Waymo_Papandreou.pdf.

[9] NAVYA, Providing Fluid Mobility with Autonomous Shuttles, NAVYA, Lyon, France, 2019, https://navya.tech/wp-content/ uploads/documents/Brochure_Shuttle_EN.pdf.

[10] L. Zhu, V. Garikapati, Y. Chen, Y. Hou, H. A. Aziz, and S. Young, "Quantifying the mobility and energy benefits of automated mobility districts using microscopic traffic simulation," in Proceedings of the International Conference on Transportation and Development 2018: Connected and Autonomous Vehicles and Transportation Safety, pp. 98-108, American Society of Civil Engineers, Reston, VA, USA, July 2018.

[11] Y. Huang, K. M. Kockelman, V. Garikapati, L. Zhu, and S. Young, "Use of shared automated vehicles for first-mile last-mile service: micro-simulation of rail-transit connections in Austin, Texas," Transportation Research Record, vol. 2675, no. 2, 2020.

[12] D. J. Fagnant and K. M. Kockelman, "The travel and environmental implications of shared autonomous vehicles, using agent-based model scenarios," Transportation Research Part C: Emerging Technologies, vol. 40, pp. 1-13, 2014.

[13] M. Maciejewski and J. Bischoff, Congestion Effects of Autonomous Taxi Fleets, Vilnius Gediminas Technical University, Vilnius, Lithuania, 2016, https://depositonce.tu-berlin. $\mathrm{de} / /$ handle/11303/8559.

[14] K. Spieser, K. Treleaven, R. Zhang, E. Frazzoli, D. Morton, and M. Pavone, "Toward a systematic approach to the design and evaluation of automated mobility-on-demand systems: a case study in Singapore," in Road Vehicle Automation, pp. 229245, Springer, Berlin, Germnay, 2014.

[15] D. J. Fagnant, K. M. Kockelman, and P. Bansal, "Operations of shared autonomous vehicle fleet for Austin, Texas, market," Transportation Research Record, vol. 2563, no. 1, pp. 98-106, 2015.

[16] M. Hyland and H. S. Mahmassani, "Dynamic autonomous vehicle fleet operations: Optimization-based strategies to assign AVs to immediate traveler demand requests," Transportation Research Part C: Emerging Technologies, vol. 92, pp. 278-297, 2018.

[17] T. D. Chen, K. M. Kockelman, and J. P. Hanna, "Operations of a shared, autonomous, electric vehicle fleet: implications of vehicle \& charging infrastructure decisions," Transportation Research Part A: Policy and Practice, vol. 94, pp. 243-254, 2016.

[18] T. D. Chen and K. M. Kockelman, "Management of a shared autonomous electric vehicle fleet: implications of pricing schemes," Transportation Research Record: Journal of the Transportation Research Board, vol. 2572, no. 1, pp. 37-46, 2016.

[19] A. Stocker and S. Shaheen, "Shared automated vehicles: Review of business models," 2017, https://www.itf-oecd.org/ shared-automated-vehicles-review-business-models.

[20] A. R. Alozi and K. Hamad, "Quantifying impacts of connected and autonomous vehicles on traffic operation using microsimulation in Dubai, UAE," in Proceedings of the 5th International Conference on Vehicle Technology and Intelligent Transport, pp. 528-535, May 2019, https://pdfs.semanticscholar.org/ 22dd/a5098e45c63b2644dacf2e1d74973362fc31.pdf.

[21] X. Guo, Y. Peng, S. Ashraf, and M. W. Burris, "Performance analyses of information-based managed lane choice decisions in a connected vehicle environment," Transportation Research Record, vol. 2674, no. 11, pp. 120-133, 2020. 
[22] L. Merlin, "Comparing automated shared taxis and conventional bus transit for a small city," Journal of Public Transportation, vol. 20, no. 2, pp. 19-39, 2017.

[23] H. K. Pinto, M. F. Hyland, H. S. Mahmassani, and I. Ö. Verbas, "Joint design of multimodal transit networks and shared autonomous mobility fleets," Transportation Research Part C: Emerging Technologies, vol. 113, 2019.

[24] Y. Shen, H. Zhang, and J. Zhao, "Integrating shared autonomous vehicle in public transportation system: a supply-side simulation of the first-mile service in Singapore," Transportation Research Part A: Policy and Practice, vol. 113, pp. 125-136, 2018.

[25] J. Wen, Y. X. Chen, N. Nassir, and J. Zhao, "Transit-oriented autonomous vehicle operation with integrated demandsupply interaction," Transportation Research Part C: Emerging Technologies, vol. 97, pp. 216-234, 2018.

[26] D. Krajzewicz, J. Erdmann, M. Behrisch, and L. Bieker, "Recent development and applications of SUMO-Simulation of Urban mobility," International Journal On Advances in Systems and Measurements, vol. 5, no. 3\&4, 2012.

[27] J. Barr, J. Beveridge, C. Clayton et al., Designing Bus Rapid Transit Running Ways, American Public Transportation Association, Washington, DC., USA, 2020, https://nacto.org/ wp-content/uploads/2016/05/2-7_APTA-Designing-BusRapid-Transit-Running-Ways_2010.pdf.

[28] J. Walker, Human Transit: How Clearer Thinking about Public Transit Can Enrich Our Communities and Our Lives, Island Press, Lahaina, HI, USA, 2012.

[29] J. Erdmann, "SUMO's lane-changing model," in Modeling Mobility with Open Data, pp. 105-123, Springer, Cham, Switzerland, 2015.

[30] Ford Motor Company, 2019 Transit: Passenger Van and Cargo Van, Ford Motor Company, Dearborn, MI, USA, 2018, https://www.ford.com/commercial-trucks/transit-cargo-van/ brochures/.

[31] GOGO Charters, Minibus Comparison Chart, GOGO Charters, Atlanta, Georgia, 2020, https://gogocharters.com/minibus-comparison-chart.

[32] I. Bae, J. Moon, and J. Seo, "Toward a comfortable driving experience for a self-driving shuttle bus," Electronics, vol. 8, no. 9, p. 943, 2019.

[33] S. Krauß, "Microscopic modeling of traffic flow: Investigation of collision free vehicle dynamics," 1998.

[34] S. Jara-Díaz and A. Tirachini, "Urban bus transport: open all doors for boarding," Journal of Transport Economics and Policy, vol. 47, no. 1, pp. 91-106, 2013.

[35] T. Litman, Autonomous Vehicle Implementation Predictions, Victoria Transport Policy Institute, Victoria, Canada, 2017.

[36] K. Kockelman and S. Boyles, Smart Transport for Cities \& Nations: The Rise of Self-Driving \& Connected Vehicles, The University of Texas at Austin, Austin, TX, USA, 2018.

[37] J. Liu, K. M. Kockelman, P. M. Boesch, and F. Ciari, "Tracking a system of shared autonomous vehicles across the Austin, Texas network using agent-based simulation," Transportation, vol. 44, no. 6, pp. 1261-1278, 2017.

[38] R. Z. Koshy and V. T. Arasan, "Influence of bus stops on flow characteristics of mixed traffic," Journal of Transportation Engineering, vol. 131, no. 8, pp. 640-643, 2005.

[39] J. Zhang, Z. Li, F. Zhang et al., "Evaluating the impacts of bus stop design and bus dwelling on operations of multitype road users," Journal of Advanced Transportation, vol. 2018, Article ID 4702517, 2018.

[40] P. Mirchandani and L. Head, "A real-time traffic signal control system: architecture, algorithms, and analysis,"
Transportation Research Part C: Emerging Technologies, vol. 9, no. 6, pp. 415-432, 2001.

[41] R. A. Retting and M. A. Greene, "Influence of traffic signal timing on red-light running and potential vehicle conflicts at urban intersections," Transportation Research Record: Journal of the Transportation Research Board, vol. 1595, no. 1, pp. 1-7, 1997.

[42] Delaware Valley Regional Planning Commission (DVRPC), SEPTA Bus Stop Design Guidelines, Delaware Valley Regional Planning Commission (DVRPC), Philadelphia, PA, USA, 2012, https://nacto.org/docs/usdg/septa_bus_stop_design_ guidelines_delaware_valley.pdf.

[43] L. He, Q. Liang, and S. Fang, "Challenges and innovative solutions in urban rail transit network operations and management: China's Guangzhou metro experience," Urban Rail Transit, vol. 2, no. 1, pp. 33-45, 2016.

[44] J. Y. Chow, S. Rath, G. Yoon, P. Scalise, and S. A. Saenz, "Spectrum of public transit operations: from fixed route to microtransit," 2020, https://c2smart.engineering.nyu.edu/wpcontent/uploads/2020/04/Chow-FTA-Report-NY-2019-06901-00.pdf. 